\title{
On Two Birkhoff-Type Interpolations with First- and Second-Order Derivative
}

\author{
Tinggang Zhao, Yongjun Li \\ School of Mathematics, Lanzhou City University, Lanzhou, China \\ Email: 13669397938@163.com, li_liyong120@163.com
}

Received 5 May 2016; accepted 12 July 2016; published 15 July 2016

\begin{abstract}
In this paper, we consider two interpolations of Birkhoff-type with integer-order derivative. The Birkhoff interpolation is related with collocation method for the corresponding initial or boundary value problems of differential equations. The solvability of the interpolation problems is proved. For Gauss-type interpolating points, error of interpolation approximation is deduced. Also, we give efficient algorithms to implement the concerned interpolations.
\end{abstract}

\section{Keywords}

Birkhoff Interpolation, Collocation Method, Gauss-Type Interpolating Point, Error Estimation

\section{Introduction}

Back to 1906, an extension of polynomial interpolation which involves the values of derivatives of the interpolated function is studied by G. D. Birkhoff in [1]. The Birkhoff interpolation (named after G. D. Birkhoff) may be defined as follow. Given a set of distinct interpolating points $\left\{x_{j}\right\}_{j=0}^{\mathbb{N}}$ and $K+1$ data $\left\{y_{j}^{m}\right\}_{j=0}^{\mathbb{N}}(K \geq N)$, to find a polynomial $p(x)$ of degree $K$ such that

$$
p^{(m)}\left(x_{j}\right)=y_{j}^{m} .
$$

If for each $j$, the orders of derivatives in (1) form a unbroken sequence, $m=0,1, \ldots, m_{j}$. The interpolation problem above refers to Hermite interpolation. But in general, we say the Birkhoff interpolation which means the other case. It turns out that the Birkhoff interpolation problem is difficult and causes many literatures [2]-[7]. In contrast to Lagrange interpolation and Hermite interpolation, a Birkhoff interpolation problem does not always have a unique solution. For instance, there is no quadratic polynomial $p$ such that $p(-1)=p(1)=0$ an $p^{\prime}(0)=1$. For solvability of Birkhoff interpolation problems, Pòlya condition is suggested in [8] and developed in [9]. To construct the Birkhoff interpolation formulation, an algorithmic approach is presented in [10] for Hermite-Birkhoff interpolation. There are also many results for $(0,2)$ interpolation [11] and others.

In any way, the approximation theory of interpolation is in foundational importance of numerical analysis even of algorithm method for various scientific computing problems. In [12], authors suggested a well-conditioned collocation method based on a Birkhoff interpolation which achieved efficiency in computation. It is necessary to contemplate the Birkhoff interpolation appeared in [12]. 
This paper is organized as follow. In Section 2, we present the interpolation problems. In Section 3, we give solvability and representation of the problems. In Section 4, we present the interpolation errors. In Section 5, efficient algorithms for computing the interpolation polynomials are given. In Section 6, some conclusive remarks are given.

\section{Interpolation Problems}

Let $\left\{x_{j}\right\}_{j=0}^{\mathbb{N}}$ be a set of $N+1$ distinct interpolating points posed on $[-1,1]$, which are arranged in increasingly order, namely

$$
-1=x_{0}<x_{1}<\ldots<x_{\mathbb{N}}=1 \text {. }
$$

Denote by $\mathbb{P}_{\mathbb{N}}$ the set of all polynomials of degree at most $N$ defined on [-1,1]. First of all, we give two definitions of the concerned Birkhoff problems.

Problem 1. For any $u \in C[-1,1]$ and $u^{\prime} \in C(-1,1]$, the Birkhoff interpolation of $u$, denoted by $I_{N}^{(1)} u$, is a polynomial of degree $N$, which satisfies that

$$
\left(I_{N}^{(1)} u\right)^{\prime}\left(x_{j}\right)=u^{\prime}\left(x_{j}\right), 1 \leq j \leq N \text {, and }\left(I_{N}^{(1)} u\right)(-1)=u(-1) .
$$

Remark. A similar interpolation for endpoint $x=1$ can be defined by conditions as

$$
\left(I_{N}^{(1)} u\right)^{\prime}\left(x_{j}\right)=u^{\prime}\left(x_{j}\right), 0 \leq j \leq N-1 \text {, and }\left(I_{N}^{(1)} u\right)(1)=u(1) .
$$

The corresponding results is parallel to theirs for Problem 1.

Problem 2. For any $u \in C[-1,1]$ and $u^{\prime \prime} \in C(-1,1)$, the Birkhoff interpolation of $u$, denoted by $I_{N}^{(2)} u$, is a polynomial of degree $N$, which satisfies that

$$
\left(I_{N}^{(2)} u\right)^{\prime \prime}\left(x_{j}\right)=u^{\prime \prime}\left(x_{j}\right), 1 \leq j \leq N-1 \text {, and }\left(I_{N}^{(2)} u\right)( \pm 1)=u( \pm 1) .
$$

We will verify that the two interpolation problems above are all solvable latterly. In common, it is unexpected that the interpolation $I_{N}^{(1)} u$ or $I_{N}^{(2)} u$ gives a good approximation to $u$ for arbitrary interpolating points. In order to achieve good approximation, we will consider the Gauss-type quadrature nodes as the interpolating points. However, solvability of the problems above has nothing to do with the interpolating points.

\section{Solvability and Representations}

\subsection{Solvability of the Problems}

For Problem 1, the solvability is equivalent to invertibility of the following matrix

$$
\left(\begin{array}{ccccccc}
1 & x_{0} & x_{0}^{2} & \cdots & x_{0}^{N-2} & x_{0}^{N-1} & x_{0}^{N} \\
0 & 1 & 2 x_{1} & \cdots & (N-2) x_{1}^{N-3} & (N-1) x_{1}^{N-2} & N x_{1}^{N-1} \\
0 & 1 & 2 x_{2} & \cdots & (N-2) x_{2}^{N-3} & (N-1) x_{2}^{N-2} & N x_{2}^{N-1} \\
\vdots & \vdots & \vdots & \ddots & \vdots & \vdots & \vdots \\
0 & 1 & 2 x_{N-1} & \cdots & (N-2) x_{N-1}^{N-3} & (N-1) x_{N-1}^{N-2} & N x_{N-1}^{N-1} \\
0 & 1 & 2 x_{N} & \cdots \in & (N-2) x_{N}^{N-3} & (N-1) x_{N}^{N-2} & N x_{N}^{N-1}
\end{array}\right)
$$

We now check the matrix (4). The determinant of the matrix (4) is $N ! \prod_{0<i<j \leq \mathbb{N}}\left(x_{i}-x_{j}\right)$. We claim that the

Problem 1 is solvable from the invertibility of the matrix (4).

For Problem 2, the solvability is equivalent to invertibility of the following matrix

$$
\left(\begin{array}{ccccccc}
1 & x_{0} & x_{0}^{2} & x_{0}^{2} & \cdots & x_{0}^{N-1} & x_{0}^{N} \\
1 & x_{N} & x_{N}^{2} & x_{N}^{2} & \cdots & x_{N}^{N-1} & x_{N}^{N} \\
0 & 0 & 2 \cdot 1 & 3 \cdot 2 x_{1} & \cdots & (N-1)(N-2) x_{2}^{N-3} & N(N-1) x_{2}^{N-2} \\
\vdots & \vdots & \vdots & \ddots & \vdots & \vdots & \vdots \\
0 & 0 & 2 \cdot 1 & 3 \cdot 2 x_{N-1} & \cdots & (N-1)(N-2) x_{N-1}^{N-3} & N(N-1) x_{N-1}^{N-2} \\
0 & 0 & 2 \cdot 1 & 3 \cdot 2 x_{N} & \cdots & (N-1)(N-2) x_{N}^{N-3} & N(N-1) x_{N}^{N-2}
\end{array}\right)
$$


We now check the matrix (5). By calculus, we know that the determinant of the matrix (5) is $2 N !(N-1) ! \prod_{0<i<j<\mathbb{N}}\left(x_{i}-x_{j}\right)$. Then Problem 2 is solvable from the invertibility of the matrix (5).

\subsection{Representations of the Interpolation Operators}

As with the Lagrange interpolation, we search for a nodal basis to represent the interpolating polynomial. For Problem 1, we need to look for $B_{j}^{(1)}(x) \in \mathbb{P}_{N}(0 \leq j \leq N)$ such that

$$
\begin{gathered}
B_{0}^{(1)}(-1)=1, \quad B_{0}^{(1)}\left(x_{i}\right)=0, \quad 1 \leq i \leq N, \\
B_{j}^{(1)}(-1)=0, \quad B_{j}^{(1)}\left(x_{i}\right)=\delta_{i j}, \quad 1 \leq i \leq N, \quad 1 \leq j \leq N .
\end{gathered}
$$

Theorem 1. The Birkhoff nodal basis has the following formulas:

$$
\begin{gathered}
B_{0}^{(1)}(x)=1, \\
B_{j}^{(1)}(x)=\int_{-1}^{x} h_{j}(t) d t, \quad 1 \leq j \leq N,
\end{gathered}
$$

where

$$
h_{j}(x)=\prod_{1 \leq k \leq N, k \neq j}\left(\frac{x-x_{k}}{x_{j}-x_{k}}\right) .
$$

Proof. The proof is direct by computing. Here we omit it.

Making use of the nodal basis in Theorem 1 , we have the representation of $I_{N}^{(1)} u(x)$ as

$$
I_{N}^{(1)} u(x)=u(-1)+\sum_{j=1}^{N} u^{\prime}\left(x_{j}\right) B_{j}^{(1)}(x) .
$$

We now turn to Problem 2. Here need to look for $B_{j}^{(2)}(x) \in \mathbb{P}_{N}(0 \leq j \leq N)$ such that

$$
\begin{gathered}
B_{0}^{(2)}(-1)=1, \quad B_{0}^{(2)} "\left(x_{i}\right)=0, \quad 1 \leq i \leq N-1, \quad B_{0}^{(2)}(1)=0, \\
B_{j}^{(2)}(-1)=0, \quad B_{j}^{(2)} "\left(x_{i}\right)=\delta_{i j}, \quad 1 \leq i \leq N-1, B_{j}^{(2)}(1)=0, \quad 1 \leq j \leq N-1, \\
B_{N}^{(2)}(-1)=0, \quad B_{N}^{(2)} "\left(x_{i}\right)=0, \quad 1 \leq i \leq N-1, \quad B_{N}^{(2)}(1)=1,
\end{gathered}
$$

Theorem 2. The Birkhoff nodal basis has the following formulas:

$$
\begin{gathered}
B_{0}^{(2)}(x)=\frac{1-x}{2}, \quad B_{N}^{(2)}(x)=\frac{1+x}{2}, \\
B_{j}^{(2)}(x)=\frac{1+x}{2} \int_{-1}^{1}(t-1) k_{j}(t) d t+\int_{-1}^{x}(x-t) k_{j}(t) d t, \quad 1 \leq j \leq N-1,
\end{gathered}
$$

where

$$
k_{j}(x)=\prod_{1 \leq k \leq N-1, k \neq j}\left(\frac{x-x_{k}}{x_{j}-x_{k}}\right) .
$$

Proof. The proof is in [12].

Making use of the nodal basis in Theorem 2, we have the representation of $I_{N}^{(2)} u(x)$ as

$$
I_{N}^{(2)} u(x)=u(-1) B_{0}^{(2)}(x)+\sum_{j=1}^{N-1} u^{\prime \prime}\left(x_{j}\right) B_{j}^{(2)}(x)+u(1) B_{N}^{(2)}(x) .
$$

\section{The Interpolation Errors on Gauss-Type Points}

In this section, we will analyze the error between the online function and its Birkhoff interpolation approximation on Gauss-type interpolating points. Let $J_{m}^{\alpha, \beta}(x)$ be the classical Jacobi orthogonal polynomial of degree 
exact $m$ with index pair $(\alpha, \beta)$. We take Jacobi-Gauss-Lobatto nodes as the interpolating points, namely, $\left\{x_{j}=x_{j, N}^{(\alpha, \beta)}\right\}_{j=0}^{N}$, and $x_{0, N}^{(\alpha, \beta)}=-1, x_{N, N}^{(\alpha, \beta)}=1, x_{j, N}^{(\alpha, \beta)}(1 \leq j \leq N-1)$ are the zeros of $\partial_{x} J_{N}^{\alpha, \beta}$ (also zeros of $J_{N-1}^{\alpha+1, \beta+1}$ ).

In order to analyze interpolation Problem 1 , we introduce the following Lagrange-Gauss-Radau interpolation $\Pi_{N-1}^{(1)}$ defined as: for $u \in C(0,1], \Pi_{N-1}^{(1)} u \in \mathbb{P}_{N-1}$, such that

$$
\Pi_{N-1}^{(1)} u\left(x_{j, N}^{(\alpha, \beta)}\right)=u\left(x_{j, N}^{(\alpha, \beta)}\right), \quad 1 \leq j \leq N .
$$

The following lemma (page 134, Theorem 3.42 in [13]) is important for error estimate.

Lemma 1. For $\alpha>-1, \beta>-2$, and any $u \in B_{\alpha, \beta+1}^{m}(-1,1)$, we have that for $0 \leq l \leq m$ and $\beta+2<m \leq N$,

$$
\left\|\partial_{x}^{l}\left(\Pi_{N-1}^{(1)} u-u\right)\right\|_{\omega^{\alpha+1, \beta+1+1}} \leq c \sqrt{\frac{(N-m) !}{(N-1) !}}(N-1)^{l-(m+1) / 2}\left\|\partial_{x}^{m} u\right\|_{\omega^{\alpha+m, \beta+m+1}},
$$

where $c$ is a positive constant independent $m, N$ and $u$.

From the Definitions of two interpolation operators $I_{N}^{(1)}$ and $\Pi_{N-1}^{(1)}$, we find that

$$
\left(I_{N}^{(1)} u\right)^{\prime}(x)=\left(\Pi_{N-1}^{(1)} u^{\prime}\right)(x) \in \mathbb{P}_{N-1} .
$$

Now, we come to the following error estimation by combining the result in Lemma 1 with (10).

Theorem 3. Under the assumption of Lemma 1, it holds

$$
\left\|\partial_{x}^{l+1}\left(I_{N}^{(1)} u-u\right)\right\|_{\omega^{\alpha+1, \beta+l+1}} \leq c \sqrt{\frac{(N-m) !}{(N-1) !}}(N-1)^{l-(m+1) / 2}\left\|\partial_{x}^{m+1} u\right\|_{\omega^{\alpha+m, \beta+m+1}},
$$

where $c$ is a positive constant independent $m, N$ and $u$.

Next, we consider the interpolation Problem 2. We need the following Lagrange-Gauss interpolation $\Pi_{N-2}^{(2)}$ defined as: for $u \in C(0,1), \Pi_{N-2}^{(2)} u \in \mathbb{P}_{N-2}$, such that

$$
\Pi_{N-2}^{(2)} u\left(x_{j, N}^{(\alpha, \beta)}\right)=u\left(x_{j, N}^{(\alpha, \beta)}\right), \quad 1 \leq j \leq N-1 .
$$

The following lemma (page 132, Theorem 3.41 in [13]) is important for error estimate.

Lemma 2. For $\alpha, \beta>-2$, and any $u \in B_{\alpha+1, \beta+1}^{m}(-1,1)$, we have for $0 \leq l \leq m<N-1$,

$$
\left\|\partial_{x}^{l}\left(\Pi_{N-2}^{(2)} u-u\right)\right\|_{\omega^{\alpha+1+1, \beta+1+1}} \leq c \sqrt{\frac{(N-m-1) !}{(N-2) !}}(N+m-2)^{l-(m+1) / 2}\left\|\partial_{x}^{m} u\right\|_{\omega^{\alpha+m+1, \beta+m+1}},
$$

where $c$ is a positive constant independent $m, N$ and $u$.

From the Definitions of two interpolation operators $I_{N}^{(2)}$ and $\Pi_{N-2}^{(2)}$ we find that

$$
\left(I_{N}^{(2)} u\right)^{\prime \prime}(x)=\left(\Pi_{N-2}^{(2)} u^{\prime \prime}\right)(x) \in \mathbb{P}_{N-2} .
$$

Now we come to the following error estimation. Similarly, combining the result in Lemma 2 with (12), we obtain

Theorem 4. Under the assumption of Lemma 2, it holds

$$
\left\|\partial_{x}^{l+2}\left(I_{N}^{(2)} u-u\right)\right\|_{\omega^{\alpha+l+1, \beta+l+1}} \leq c \sqrt{\frac{(N-m-1) !}{(N-2) !}}(N+m-2)^{l-(m+1) / 2}\left\|\partial_{x}^{m+2} u\right\|_{\omega^{\alpha+m+1, \beta+m+1}},
$$

where $c$ is a positive constant independent $m, N$ and $u$.

\section{Efficient Formulations to Computing the Interpolations}

The representation (7) (or (9)) with Theorem 1 (or with Theorem 2) is unconvenience for computing $I_{N}^{(1)}$ (or $\left.I_{N}^{(2)}\right)$. Here, we develop efficient algorithms to deal with them.

Case $I_{N}^{(1)}$. Since $I_{N}^{(1)} u(x) \in \mathbb{P}_{N}$, we write

$$
I_{N}^{(1)} u(x)=\sum_{l=0}^{N} a_{l} J_{l}^{\alpha-1, \beta}(x) .
$$

We only need to compute $a_{l}(0 \leq l \leq N)$. Derivating (14), we have 


$$
\left(I_{N}^{(1)} u\right)^{\prime}(x)=\sum_{l=1}^{N} a_{l} \frac{l+\alpha+\beta}{2} J_{l-1}^{\alpha, \beta+1}(x) .
$$

Making use of the orthogonality of Jacobi polynomials, we can obtain for $1 \leq l \leq N$,

$$
a_{l}=\frac{2}{(1+\alpha+\beta) \gamma_{l-1}^{(\alpha, \beta+1)}} \int_{-1}^{1}\left(I_{N}^{(1)} u\right)^{\prime}(x) J_{l-1}^{\alpha, \beta+1}(x) \omega^{\alpha, \beta+1}(x) d x,
$$

Since $\left(I_{N}^{(1)} u\right)^{\prime}(x) J_{l-1}^{\alpha, \beta+1}(x) \in \mathbb{P}_{2 N-2}$, the Jacobi-Gauss-Radau quadrature at nodes $x_{j}(1 \leq j \leq N)$ is exact. At last, we have

$$
a_{l}=\frac{2}{(1+\alpha+\beta) \gamma_{l-1}^{(\alpha, \beta+1)}} \sum_{j=1}^{N}\left(I_{N}^{(1)} u\right)^{\prime}\left(x_{j}\right) J_{l-1}^{\alpha, \beta+1}\left(x_{j}\right) \omega_{j},
$$

where $\omega_{j}(1 \leq j \leq N)$ is the weight corresponding to quadrature node $x_{j}$ of Jacobi-Gauss-Radau with the fixed endpoint $x=1$. Now we only left $a_{0}$ to determine. It is not difficult because that

$$
a_{0}=u(-1)-\sum_{l=1}^{N} a_{l} J_{l}^{\alpha-1, \beta}(-1)=u(-1)-\sum_{l=1}^{N}(-1)^{l} a_{l} \frac{\Gamma(l+\beta+1)}{l ! \Gamma(\beta+1)} .
$$

Case $I_{N}^{(2)}$. Since $I_{N}^{(2)} u(x) \in \mathbb{P}_{N}$, we write

$$
I_{N}^{(2)} u(x)=\sum_{l=0}^{N} a_{l} J_{l}^{\alpha-1, \beta-1}(x) .
$$

We need to compute $a_{l}(0 \leq l \leq N)$. Derivating (18) twice, we have

$$
\left(I_{N}^{(2)} u\right)^{\prime \prime}(x)=\sum_{l=2}^{N} a_{l} \frac{(l+\alpha+\beta-1)(l+\alpha+\beta)}{2^{2}} J_{l-2}^{\alpha+1, \beta+1}(x) .
$$

Making use of the orthogonality of Jacobi polynomials, we can obtain for $2 \leq l \leq N$,

$$
a_{l}=\frac{2^{2}}{(l+\alpha+\beta-1)(l+\alpha+\beta) \gamma_{l-2}^{(\alpha+1, \beta+1)}} \int_{-1}^{1}\left(I_{N}^{(2)} u\right)^{\prime \prime}(x) J_{l-2}^{\alpha+1, \beta+1}(x) \omega^{\alpha+1, \beta+1}(x) d x .
$$

Since $\left(I_{N}^{(2)} u\right)^{\prime \prime}(x) J_{l-2}^{\alpha+1, \beta+1}(x) \in \mathbb{P}_{2 N-4}$, the Jacobi-Gauss quadrature at nodes $x_{j}(1 \leq j \leq N-1)$ is exact.

At last, we have for $2 \leq l \leq N$

$$
a_{l}=\frac{2^{2}}{(l+\alpha+\beta-1)(l+\alpha+\beta) \gamma_{l-2}^{(\alpha+1, \beta+1)}} \sum_{j=1}^{N-1}\left(I_{N}^{(2)} u\right)^{\prime \prime}\left(x_{j}\right) J_{l-2}^{\alpha+1, \beta+1}\left(x_{j}\right) \varpi_{j},
$$

where $\varpi_{j}(1 \leq j \leq N-1)$ is the weight corresponding to quadrature node $x_{j}$ of Jacobi-Gauss. Now there left $a_{0}$ and $a_{1}$ to be determined. In fact,

$$
a_{1}=\frac{u(1)-u(-1)-\sum_{l=2}^{N} a_{l}\left(J_{l}^{\alpha-1, \beta-1}(1)-J_{l}^{\alpha-1, \beta-1}(-1)\right)}{J_{1}^{\alpha-1, \beta-1}(1)-J_{1}^{\alpha-1, \beta-1}(-1)} .
$$

and

$$
a_{0}=u(-1)-\sum_{l=1}^{N} a_{l} J_{l}^{\alpha-1, \beta-1}(-1) .
$$

For every $0 \leq l \leq N$, we need $O(N)$ operations to computing $a_{l}$ in both cases. The computational complexity is $O\left(N^{2}\right)$ to obtain the interpolation polynomial (14) or (18) assuming that $\gamma_{j}^{\alpha, \beta+1}, J_{l}^{\alpha, \beta}\left(x_{j}\right), \omega_{j}$ or $\varpi_{j}$ are all pre-computed.

\section{Conclusive Remarks}

Two Birkhoff-type interpolations which are related with first-order initial value problem and second-order 
boundary value problem respectively are considered in the paper. As in [12], this Birkhoff interpolation leads to well-conditioned collocation matrices or pre-conditioner. We remark that it is worthy to consider the similar interpolations for high-order derivative case or fractional-order derivative case.

\section{Acknowledgements}

We thank the editor and the referee for their comments. The authors were supported by National Natural Science Foundation of China (Grant No. 11161026 and No. 11261027).

\section{References}

[1] Birkhoff, G.D. (1906) General Mean Value and Remainder Theorems with Applications to Mechanical Differentiation and Quadrature. Transactions of the American Mathematical Society, 7, 107-136. http://dx.doi.org/10.2307/1986339

[2] Lorentz, G.G. and Zeller, K.L. (1971) Birkhoff Interpolation. SIAM Journal on Numerical Analysis, 8, 43-48. http://dx.doi.org/10.1137/0708006

[3] Lorentz, G.G., Jetter, K. and Riemenschneider, S.D. (1983) Birkhoff Interpolation. Addison-Wesley Publ. Comp.

[4] Pólya, G. (1931) Bemerkung zur interpolation und zur Näherungstheorie der dalkenbiegung. Zeitschrift für Angewandte Mathematik und Mechanik, 11, 445-449. http://dx.doi.org/10.1002/zamm.19310110620

[5] Karlin, S. and Karon, J.M. (1972) On Hermite-Birkhoff Interpolation. Journal of Approximation Theory, 6, 90-114. http://dx.doi.org/10.1016/0021-9045(72)90085-8

[6] Sharma, A. (1972) Some Poised and Unpoised Problems of Interpolation. SIAM Review, 14, 129-151. http://dx.doi.org/10.1137/1014004

[7] Shi, Y.G. (2003) Theory of Birkhoff Interpolation. Nova Science Publishers, New York.

[8] Ferguson, D.R. (1969) The Question of Uniqueness for G. D. Birkhoff Interpolation Problems. Journal of Approximation Theory, 2, 1-28. http://dx.doi.org/10.1016/0021-9045(69)90028-8

[9] Palacios, F. and Rubió, P. (2003) Generalised Pólya Condition for Birkhoff Interpolation with Lacunary Polynomial. Applied Mathematics E-Notes, 3, 124-129.

[10] Mühlbach, G. (1981) An Algorithm Approach to Hermite-Birkhoff Interpolation. Numerische Mathematik, 37, 339347. http://dx.doi.org/10.1007/BF01400313

[11] Szili, L. (1996) A Survey on (0,2) Interpolation. Annales Universitatis Scientiarum Budapestinensis de Rolando Eötvös Nominatae, Sectio Computatorica, 16, 377-390.

[12] Wang, L.L., Samson, M.D. and Zhao, X.D. (2014) A Well-Conditioned Collocation Method Using a Pseudospectral Integration Matrix. SIAM: SIAM Journal on Scientific Computing, 36, A907-A929. http://dx.doi.org/10.1137/130922409

[13] Shen, J., Tang, T. and Wang, L.L. (2011) Spectral Methods: Algorithms, Analysis and Applications. Springer, Berlin. http://dx.doi.org/10.1007/978-3-540-71041-7

\section{Submit or recommend next manuscript to SCIRP and we will provide best service for you:}

Accepting pre-submission inquiries through Email, Facebook, LinkedIn, Twitter, etc.

A wide selection of journals (inclusive of 9 subjects, more than 200 journals)

Providing 24-hour high-quality service

User-friendly online submission system

Fair and swift peer-review system

Efficient typesetting and proofreading procedure

Display of the result of downloads and visits, as well as the number of cited articles

Maximum dissemination of your research work

Submit your manuscript at: http://papersubmission.scirp.org/ 